(2) Open Access Full Text Article

REVIEW

\title{
Dasatinib for the treatment of chronic myeloid leukemia: patient selection and special considerations
}

This article was published in the following Dove Press journal:

Drug Design, Development and Therapy

13 October 2016

Number of times this article has been viewed

\section{Dilek Keskin}

Sevil Sadri

Ahmet Emre Eskazan

Department of Internal Medicine, Division of Hematology, Cerrahpasa Faculty of Medicine, Istanbul University, Istanbul, Turkey
Correspondence: Ahmet Emre Eskazan Department of Internal Medicine, Division of Hematology, Cerrahpasa Faculty of Medicine, Istanbul University, Kocamustafapasa/Fatih, Istanbul 34303, Turkey

Tel +90 5337227376

Fax +90 212 5897934

Email emreeskazan@hotmail.com
Abstract: Dasatinib is one of the second-generation tyrosine kinase inhibitors used in imatinib resistance and/or intolerance, as well as in the frontline setting in patients with chronic myeloid leukemia-chronic phase, and also in patients with advanced disease. It is also utilized in Philadelphia chromosome-positive acute lymphocytic leukemia. While choosing the appropriate tyrosine kinase inhibitor (ie, dasatinib) for each individual patient, comorbidities and $B C R-A B L 1$ kinase domain mutations should always be taken into consideration, among other things. This review mainly focuses on patient selection prior to dasatinib administration in the treatment of chronic myeloid leukemia.

Keywords: chronic myeloid leukemia, comorbidity, dasatinib, mutation, tyrosine kinase inhibitor

\section{Introduction}

Chronic myeloid leukemia (CML) is a myeloproliferative neoplasm with an annual incidence of $0.6-2$ cases per 100,000 adults in US and Europe and is responsible for $15 \%$ of newly diagnosed cases of leukemia in adults. ${ }^{1,2}$ Median age at diagnosis is 60-65 years according to most epidemiological registries. The incidence of CML is slightly higher in males than in females. CML is characterized by a Philadelphia chromosome (Ph), which results in the formation of the $B C R-A B L 1$ fusion gene. ${ }^{1}$ Eighty-five percent of patients present with chronic-phase CML (CML-CP), while the rest present with advanced disease stages (10\% in accelerated phase [AP] and approximately $5 \%$ in blast crisis $[\mathrm{BC}])$. In the natural course of the disease, most of the patients transform to AP before moving into $\mathrm{BC}$, but $20 \%$ of the patients transform to $\mathrm{BC}$ without prior $\mathrm{AP}{ }^{2}$

Tyrosine kinase inhibitors (TKIs) are currently the mainstay of CML treatment, and the first approved TKI in the treatment of CML was imatinib mesylate. Although second-generation TKIs (2GTKIs) (nilotinib and dasatinib) can be administered in the frontline setting in some countries, imatinib is widely accepted to be the first-line treatment option in patients with CML-CP. ${ }^{3}$ Imatinib has revolutionized the treatment of CML, but primary/secondary resistance as well as intolerance may occur. ${ }^{4,5}$ Resistance to TKIs may arise from various mechanisms, including decreasing intracellular drug levels, increasing expression of $B C R-A B L 1$, mutations in the ABL kinase affecting drug interaction or kinase activity, and $B C R$ - $A B L 1$-independent mechanisms. ${ }^{4}$ In patients with resistance and/or intolerance to imatinib, 2GTKIs can be utilized, which then brings forth the question "Which TKI for which patient?". Physicians should mainly consider the comorbidities and $B C R-A B L 1$ mutations while starting a TKI treatment in a patient with imatinib resistance. In this review, we mainly focus 
on the patient selection prior to dasatinib administration in the treatment of CML.

\section{Dasatinib}

\section{Structure, mechanism, pharmacokinetics, and pharmacogenetics}

Originally termed BMS-354825, dasatinib (Sprycel ${ }^{\circledR}$; Bristol-Myers Squibb, New York, NY, USA) is an orally potent, bioavailable inhibitor of $B C R-A B L 1$ and was approved by the US Food and Drug Administration (FDA) in 2006 for the treatment of imatinib-resistant and -intolerant adults with CML-CP and advanced disease as well as Ph-positive acute lymphoblastic leukemia. ${ }^{6,7}$ It is largely metabolized in the liver, mainly by the cytochrome P450 isoenzyme CYP3A4. As a result, there is potential for various drug-drug interactions (eg, when dasatinib is coadministered with a drug which also prolongs QTc and/or concomitant administration of dasatinib with CYP3A4 inhibitors or inducers). Therefore, if possible, these combinations should be avoided; however, if coadministration is inevitable, a dose adjustment may be warranted, and strict monitoring for toxicity and efficacy is necessary. ${ }^{8}$ The incomplete oral bioavailability of dasatinib may be low due to poor absorption from the gastrointestinal tract and/or high first-pass metabolism. ${ }^{9}$ Furthermore, the solubility of dasatinib is pH-dependent, and long-term inhibition of gastric acid secretion reduces dasatinib exposure. ${ }^{10,11}$

In addition to blocking $B C R-A B L 1$ kinase activity, dasatinib inhibits a distinct spectrum of oncogenic kinases, including Src family kinases (SFKs), c-Kit, platelet-derived growth factor-receptor (PDGFR), and ephrin-A receptor. ${ }^{7,10,12}$ Because dasatinib is not a substrate for organic cation transporter-1, it potently inhibits SFKs, which are associated with $B C R-A B L 1$-independent imatinib resistance, which is likely to be largely responsible for low in vitro sensitivity to imatinib. Dasatinib is 325 times more potent in vitro for unmutated $B C R-A B L 1$ than imatinib. This suggests that dasatinib may overwhelm the imatinib resistance caused by increased $B C R-A B L 1$ expression. ${ }^{7,12}$ Dasatinib binds both the active and inactive forms of $B C R-A B L 1$ and has in vitro activity against all currently described imatinib-resistant mutations except T315I. It may also overcome different resistance mechanisms to imatinib, including alternate signaling pathways involving the SFKs and $M D R-1$ gene overexpression. The FDA-approved dosages are $100 \mathrm{mg}$ per day once daily orally for patients with CML-CP and $140 \mathrm{mg}$ once daily for patients with advanced disease. Additionally dosing modifications can be made based on toxicities. ${ }^{13}$

\section{Dasatinib in the treatment of CML}

Dasatinib has been first approved for the second-line treatment of CML patients who are intolerant and/or resistant to imatinib. ${ }^{5,14}$ Durable complete cytogenetic and major molecular responses can be achieved after prolonged administration of dasatinib in patients with CML-CP with a very low likelihood of disease transformation ( $<3 \%$ at 24 months). ${ }^{15,16}$

DASISION (DASatinib versus Imatinib Study In treatment-Naive CML patients) study is a randomized Phase III trial testing dasatinib $100 \mathrm{mg}$ once daily versus imatinib $400 \mathrm{mg}$ once daily in patients with newly diagnosed CML-CP. ${ }^{17,18}$ In this study, patients receiving first-line dasatinib achieved faster and deeper molecular responses, a result that was also supported by other trials. ${ }^{19,20}$ Following DASISION trial, dasatinib $100 \mathrm{mg}$ daily was approved for the upfront treatment of CML. ${ }^{5,14}$ For advanced disease, the daily dosing of dasatinib is $140 \mathrm{mg}$ which can be given as one of the following two doses: $70 \mathrm{mg}$ twice daily or $140 \mathrm{mg}$ once daily. ${ }^{5}$

That a rapid response has been shown to correlate with better long-term clinical outcomes, both in the frontline use and also after imatinib failure, ${ }^{21}$ for patients with a high Sokal score, starting treatment with either dasatinib or nilotinib can be reasonable, although this recommendation is an experts' opinion $^{21,22}$ mainly owing to on the greater potency of these agents, which has led to higher rates of early molecular response and major molecular response.

The choice between these two 2GTKIs is governed mostly by their potential toxicity profiles as well as the comorbidities of the patients. ${ }^{23,24}$ Also, the presence of $B C R-A B L 1$ kinase domain (KD) point mutations influence the choice of the second-line TKI treatment in patients with CML-CP who are resistant to prior TKI therapy. ${ }^{5,25}$ For example, in case of $\mathrm{Y} 253 \mathrm{H}, \mathrm{E} 255 \mathrm{~K} / \mathrm{V}$, or $\mathrm{F} 359 \mathrm{~V} / \mathrm{C} / \mathrm{I}$ mutations, dasatinib is probably more effective than nilotinib. ${ }^{25}$ Although in patients harboring F317L/V/I/C mutations, nilotinib should be the treatment choice, ${ }^{24}$ there can be always some exceptions described in the literature. ${ }^{26,27}$ In addition to that, in a study demonstrating real-life data on second-line dasatinib use among elderly patients (aged $>60$ years) with CML, detection of $B C R-A B L 1 \mathrm{KD}$ mutations was shown to have a negative impact on the response to dasatinib after imatinib failure. ${ }^{28}$

\section{Adverse events during dasatinib therapy}

Since the life expectancy of CML patients had increased in the era of the TKIs, CML-treating physicians are facing another important issue - quality of life (QoL). In daily 
clinical practice, intolerance to the TKIs could interfere with the QoL in patients with $\mathrm{CML},{ }^{29}$ and in order to improve QoL, monitoring for and managing adverse events (AEs) of the TKIs promptly and maintaining the patients' adherence to the TKI treatment could optimize patient outcomes..$^{23}$ The comorbidities have an impact on the selection of the TKI so as to minimize the risk of AEs and as a result maximize the adherence to TKI therapy. ${ }^{24}$

Dasatinib is generally well tolerated but can be associated with usually reversible and manageable AEs. ${ }^{13,23,29}$ Although such events commonly appear at the beginning of treatment and are predominantly mild to moderate, self-limiting or resolved with supportive care, temporary interruption, or dose reduction, some can occur in the late course of therapy which may interfere with QoL and result in quitting the drug permanently. Common hematologic and nonhematologic AEs of dasatinib are summarized in Table 1.

The frequencies of AEs may vary from study to study, depending on whether dasatinib is given upfront or after any TKI failure/intolerence, and also the rates of AEs tend to increase with higher doses (ie, patients receiving dasatinib $140 \mathrm{mg}$ daily tend to have more AEs than cases with lower doses) and in patients with advanced phase of disease as well as in elderly patients especially with comorbidities and multiple concomitant medications (eg, CYP3A4 inhibitors).

Table I Common hematologic and nonhematologic AEs of dasatinib

\begin{tabular}{ll}
\hline Hematologic AEs & Nonhematologic AEs \\
\hline Thrombocytopenia & Cardiac \\
Anemia & Pericardial effusion \\
NK/T-cell lymphocytosis & Arrhythmia-QTc prolongation \\
Neutropenia & Accelerated atherosclerosis \\
& Pulmonary \\
Pleural effusion \\
Pulmonary arterial hypertension \\
Dyspnea \\
Gastrointestinal \\
Abnormal liver enzymes \\
Hyperbilirubinemia \\
Diarrhea \\
Vomiting \\
Nausea \\
Musculosketal \\
Myalgia \\
Myositis \\
Others \\
Headache \\
Hair discoloration \\
Skin rash \\
Panniculitis \\
\end{tabular}

Abbreviation: AEs, adverse events.
In DASISION trial, where dasatinib was administered $100 \mathrm{mg}$ once daily in the frontline setting, grades III-IV hematologic AEs including neutropenia (21\%), thrombocytopenia (19\%), and anemia (10\%) were observed. ${ }^{17}$ In imatinib-resistant cases, the frequencies of hematologic AEs can be different than those occurring in the first-line setting, and in one study the rates of cytopenias were lower among CML-CP patients receiving dasatinib $100 \mathrm{mg}$ once daily relative to the patients in other treatment arms. ${ }^{25}$

In the treatment of patients who have experienced imatinib resistance or intolerance, dasatinib $70 \mathrm{mg}$ twice daily was associated with the occurrence of generally low-grade (I-II) nonhematologic AEs, the most frequent of which were fluid retention (including pleural and pericardial effusions), dyspnea, infection, headache, diarrhea, fatigue, and abdominal and musculoskeletal pain. ${ }^{30,31}$ In the study by Shah et al, ${ }^{32}$ the most common nonhematologic AEs were also nausea, headache, diarrhea, and fatigue, and treatment with $100 \mathrm{mg}$ daily resulted in lower rates of discontinuation due to drug toxicity $(11 \%)$ than treatment with $70 \mathrm{mg}$ twice daily $(21 \%)$.

Being a potent $2 \mathrm{GTKI}$ inhibiting $B C R-A B L 1, \mathrm{C}-\mathrm{KIT}$, PDGFR, and SRC pathways, ${ }^{33,34}$ patients receiving dasatinib may develop some nonhematologic AEs which are thought to be the "off-target effects" of dasatinib. Pleural effusion (PE), which has been observed in up to $14 \%-35 \%$ in patients who were resistant/intolerant to imatinib, has been related to an autoimmune-mediated mechanism or inhibition of PDGFR- $\beta$, as opposed to fluid retention. ${ }^{35,36}$ In the frontline setting, PE was seen approximately in $10 \%$ of the patients at all grades. ${ }^{17}$ Especially in patients with severe (Grades III-IV) PE under dasatinib, QoL is markedly affected, and in addition to that, the real-life data with CML patients receiving dasatinib after imatinib failure can be associated with higher incidence of PEs, than reported in clinical trials especially when the follow-up period extends. ${ }^{37}$ Even in imatinib-intolerant/-resistant CML patients who were treated with smaller doses of dasatinib (ie, $50 \mathrm{mg}$ daily), PEs may occur. ${ }^{38}$

There are several strategies that can be performed in the management of PE, including dose interruption/reduction and supportive measures, including administration of diuretics and glucocorticoids. Sometimes, invasive procedures such as thoracenteses or tube drainage are needed if grades III-IV $\mathrm{PE}$ is present. ${ }^{37}$ If PE persists or reoccurs, switching to an alternative TKI treatment should be considered.

Nilotinib might induce accelerated atherosclerosis ${ }^{39}$ and can cause peripheral artery occlusive disease in the long-term follow-up. ${ }^{40}$ However, vascular AEs can be problematic in 
patients receiving other TKIs, ${ }^{33}$ and cardiovascular AEs can also be observed in patients receiving dasatinib, especially among nonselected patients in the real-life setting. ${ }^{41}$ In a recent meta-analysis, it was shown that the risk of vascular AEs was increased with dasatinib (odds ratio $[\mathrm{OR}]=3.86$; $95 \%$ confidence interval $[\mathrm{CI}]=1.33-11.18$ ) as well as with nilotinib and ponatinib when compared to imatinib. ${ }^{42}$ Also, in a more recent population-based cohort study performed in Sweden, ${ }^{43}$ it was shown that there was an increased risk of arterial and venous vascular AEs in patients with CML treated with a TKI than in an age- and sex-matched control population. The 2GTKIs (dasatinib and nilotinib) induced arterial thromboembolic events with similar frequencies; however, there were no patients with venous thromboembolic events among those receiving dasatinib. ${ }^{43}$ So, attention should be paid to the potential cardiovascular toxicity of the TKIs, and cardiovascular risk factors must be considered and (if possible) optimized when patients with CML are being treated particularly with 2GTKIs.

Recently, pulmonary hypertension and pulmonary arterial hypertension (PAH) have been reported as infrequent but significant AEs associated with dasatinib, ${ }^{44,45}$ and these can usually be managed with dose interruption/reduction of the drug and changing the TKI. Most patients have at least one cardiovascular or pulmonary risk factor/symptom in their medical history at the time of pulmonary hypertension. Possible mechanisms behind dasatinib-induced PAH include the involvement of the immune system, ${ }^{46}$ while another possible mechanism involves the known pan-SRC kinase inhibition by dasatinib. SRC kinase is involved in the regulation of smooth muscle cell proliferation and vasoconstriction. Inhibition of SRC by dasatinib may result in proliferation of endothelial cells and pulmonary arterial smooth muscle, resulting in PAH in some patients. ${ }^{47}$ In addition to that, dasatinib-induced PAH may be an idiosyncratic reaction or it can be related to preexisting conditions or prior therapies that caused vascular damage or inflammation. ${ }^{44}$

Kim et al ${ }^{48}$ showed that natural killer (NK) or NK/T-cell lineage peripheral monoclonal large granular lymphocytosis (LGL) can be associated with dasatinib therapy in Ph-positive leukemic patients. Mustjoki and colleagues ${ }^{49}$ also demonstrated that there was a clonal expansion of T- or NK-LGL during dasatinib treatment. From the initiation of dasatinib, mean time to generation of lymphocytosis was 3 months (range, 1-15 months), and persistent and durable lymphocytosis was seen in most of the patients during dasatinib treatment. The generation of LGL during dasatinib therapy was shown to be associated with high response rates and favorable outcome. ${ }^{37,50-53}$ It was also shown that NK/T lymphocytosis, which appears in peripheral blood, was also detected in PE. ${ }^{50}$

As NK cell and $\mathrm{CD} 8^{+}$T-cell (partially) functions are regulated by killer immunoglobulin-like receptors (KIRs), Kreutzman et $\mathrm{al}^{54}$ performed a study evaluating KIR gene profile in dasatinib-treated CML patients and analyzed whether KIR gene profiles were associated with the treatment outcomes. In that study, KIR gene profile was found to be associated with clinical response in CML-CP patients receiving dasatinib. ${ }^{54}$ Although further studies are needed to better elucidate how different KIRs affect therapy response; hypothetically, KIR gene profile can be evaluated prior to TKI therapy and dasatinib can be the choice of therapy in patients with $K I R$ gene profiles which were found to be associated with a favorable outcome under dasatinib.

Lymphadenopathy with morphologic features of reactive follicular hyperplasia (RFH) was described in a cohort of patients with CML on relatively long-term dasatinib therapy. ${ }^{55,56}$ It was thought that the impact of dasatinib on SFKs and on Akt/PKB pathway could contribute to the RFH initiation. Dasatinib discontinuation should be encouraged if the diagnosis of RFH is suspected, and the lymphadenopathies usually resolve with discontinuation of therapy. ${ }^{55}$ If lymphadenopathy occurs during dasatinib therapy in a patients with CML without any additional signs/symptoms, consideration of dasatinib-related lymphadenopathy may preempt unnecessary repeat diagnostic procedures.

Bleeding episodes can occur in $20 \%-25 \%$ of patients treated with dasatinib but are grades III-IV in only $10 \%$ of cases. ${ }^{29,57}$ Although bleeding usually occurs in the setting of thrombocytopenia, in approximately one third of the cases, bleeding occurs with adequate platelet counts. ${ }^{57}$ In patients who develop bleeding on dasatinib, therapy should be interrupted and/or treatment discontinuation can be necessary in some cases. It has also been reported that dasatinib may inhibit platelet aggregation. ${ }^{58}$ Thus, concomitant administration of drugs that inhibit platelet function, such as nonsteroidal anti-inflammatory agents, is not recommended during dasatinib treatment. ${ }^{29}$

Dasatinib may also cause acute colitis and rectal bleeding both by decreasing immune tolerance against intestinal microflora by reducing immunoregulatory cells and inhibiting signal transduction pathways. ${ }^{59,60}$ Dasatinib-induced colitis usually regresses after cessation of the drug, and switching to another TKI is a reasonable intervention. If a CML patient has a history of bleeding diathesis and/or colitis, it may be wise to choose a TKI other than dasatinib in this kind of patient. 


\section{Patient selection}

The life expectancy of CML patients had increased since TKIs were introduced, and the comorbidities have an impact on the selection of the TKI. Dasatinib should be avoided for a patient with prior lung disease or gastrointestinal bleeding. ${ }^{21,23}$ In the absence of comorbidities or risk factors, the choice between these two drugs is left to patient preference for either a once per day medication (dasatinib) versus one that is taken twice per day on an empty stomach (nilotinib). Drug-drug interactions should always be taken into account, both at the treatment initiation and during the follow-up. In CML-CP patients after imatinib failure, $B C R-A B L 1 \mathrm{KD}$ point mutation testing should be performed prior to second-line TKI treatment, and dasatinib can be the treatment choice where appropriate. ${ }^{25}$ In the context of advanced disease, especially in patients with $\mathrm{BC}$, dasatinib can be the choice of TKI treatment over imatinib and nilotinib, since central nervous system involvement may be observed in $\mathrm{BC}$ and dasatinib can penetrate through the blood-brain barrier.

\section{Conclusion}

Each TKI (eg, dasatinib) is associated with a distinct safety profile that should be considered when choosing the optimal treatment for an individual with CML. While choosing a TKI treatment, comorbidities of the patient together with the possible drug-drug interactions, both prior to and during the TKI therapy, should be taken into consideration. In patients with imatinib failure, $B C R-A B L 1 \mathrm{KD}$ mutation testing should be performed while starting a second-line TKI treatment. During the follow-up, close monitoring is essential for response evaluation, as well as to detect and manage TKI-associated AEs properly.

\section{Acknowledgment}

We would like to thank Professor Teoman Soysal for his continuous support and mentoring.

\section{Disclosure}

AEE had received honorarium from Novartis. The authors report no other conflicts of interest in this work.

\section{References}

1. Gugliotta G, Castagnetti F, Apolinari M, et al. First-line treatment of newly diagnosed elderly patients with chronic myeloid leukemia: current and emerging strategies. Drugs. 2014;74(6):627-643.

2. Jabbour E, Kantarjian H. Chronic myeloid leukemia: 2014 update on diagnosis, monitoring, and management. Am J Hematol. 2014;89(5): 547-556.

3. Chen R, Chen B. The role of dasatinib in the management of chronic myeloid leukemia. Drug Des Devel Ther. 2015;9:773-779.
4. Bixby D, Talpaz M. Seeking the causes and solutions to imatinibresistance in chronic myeloid leukemia. Leukemia. 2011;25(1):7-22.

5. Baccarani M, Deininger MW, Rosti G, et al. European LeukemiaNet recommendations for the management of chronic myeloid leukemia: 2013. Blood. 2013;122(6):872-884.

6. Baker SJ, Reddy EP. Targeted inhibition of kinases in cancer therapy. Mt Sinai J Med. 2010;77(6):573-586.

7. Schenone S, Brullo C, Musumeci F, Botta M. Novel dual Src/Abl inhibitors for hematologic and solid malignancies. Expert Opin Investig Drugs. 2010;19(8):931-945.

8. Haouala A, Widmer N, Duchosal MA, Montemurro M, Buclin T, Decosterd LA. Drug interactions with the tyrosine kinase inhibitors imatinib, dasatinib, and nilotinib. Blood. 2011;117(8):e75-e87.

9. Liu F, Lang LW, Jiang J, Lu HJ, Wang JM, Wang SC. Synthesis and biopharmaceutical studies of JLTN as potential dasatinib prodrug. Chem Pharm Bull (Tokyo). 2013;61(8):877-881.

10. Condorelli F, Genazzani AA. Dasatinib: is it all in the dose? BioDrugs. 2010;24(3):157-163.

11. McFarland KL, Wetzstein GA. Chronic myeloid leukemia therapy: focus on second-generation tyrosine kinase inhibitors. Cancer Control. 2009; 16(2):132-140.

12. Stein B, Smith BD. Treatment options for patients with chronic myeloid leukemia who are resistant to or unable to tolerate imatinib. Clin Ther. 2010; 32(5):804-820.

13. Jabbour E, Deininger M, Hochhaus A. Management of adverse events associated with tyrosine kinase inhibitors in the treatment of chronic myeloid leukemia. Leukemia. 2011;25(2):201-210.

14. National Comprehensive Cancer Network. Chronic Myeloid Leukemia (Version 1.2016). Available from: http://www.nccn.org/professionals/ physician_gls/pdf/cml.pdf. Accessed February 2, 2016.

15. Shah NP, Kim DW, Kantarjian H, et al. Potent, transient inhibition of BCRABL with dasatinib $100 \mathrm{mg}$ daily achieves rapid and durable cytogenetic responses and high transformation-free survival rates in chronic phase chronic myeloid leukemia patients with resistance, suboptimal response or intolerance to imatinib. Haematologica. 2010;95(2):232-240.

16. Rea D, Vellenga E, Junghanss C, et al. Six-year follow-up of patients with imatinib-resistant or imatinib-intolerant chronic phase chronic myeloid leukemia receiving dasatinib. Haematologica. 2012;97(Suppl 1):199.

17. Kantarjian H, Shah NP, Hochhaus A, et al. Dasatinib versus imatinib in newly diagnosed chronic-phase chronic myeloid leukemia. $N$ Engl J Med. 2010;362(24):2260-2270.

18. Kantarjian HM, Shah NP, Cortes JE, et al. Dasatinib or imatinib in newly diagnosed chronic phase chronic myeloid leukemia: 2-year follow up from a randomized phase 3 trial (DASISION). Blood. 2012;119(5):1123-1129.

19. Radich JP, Kopecky KJ, Appelbaum FR, et al. A randomized trial of dasatinib $100 \mathrm{mg}$ versus imatinib $400 \mathrm{mg}$ in newly diagnosed chronic phase chronic myeloid leukemia. Blood. 2012;120(19):3898-3905.

20. Hjorth-Hansen H, Stenke L, Söderlund S, et al; Nordic CML Study Group. Dasatinib induces fast and deep responses in newly diagnosed chronic myeloid leukaemia patients in chronic phase: clinical results from a randomised phase-2 study (NordCML006). Eur J Haematol. 2015; 94(3):243-250.

21. Larson RA. Is there a best TKI for chronic phase CML? Blood. 2015; 126(21):2370-2375.

22. Keskin D, Eskazan AE. The treatment of chronic myeloid leukaemia (CML) in the era of tyrosine kinase inhibitors - what is new in the battle of CML? Eur Oncol Haematol. 2015;11(1):30-31.

23. Carneiro BA, Kaplan JB, Giles FJ. Tyrosine kinase inhibitor therapy in chronic myeloid leukemia: update on key adverse events. Expert Rev Hematol. 2015;8(4):457-479.

24. Gugliotta G, Castagnetti F, Fogli M, et al. Impact of comorbidities on the treatment of chronic myeloid leukemia with tyrosine-kinase inhibitors. Expert Rev Hematol. 2013;6(5):563-574.

25. Soverini S, Hochhaus A, Nicolini FE, et al. BCR-ABL kinase domain mutation analysis in chronic myeloid leukemia patients treated with tyrosine kinase inhibitors: recommendations from an expert panel on behalf of European LeukemiaNet. Blood. 2011;118(5):1208-1215. 
26. Eskazan AE, Soysal T, Erbilgin Y, Ozbek U, Ferhanoglu B. Chronic myeloid leukemia patients with F317L BCR-ABL kinase domain mutation are resistant to dasatinib: is that true for all the patients? Leuk Res. 2011;35(9):e145-e146.

27. Eşkazan AE, Soysal T. Dasatinib may override F317L BCR-ABL kinase domain mutation in patients with chronic myeloid leukemia. Turk J Haematol. 2013;30(2):211-213.

28. Tiribelli M, Latagliata R, Luciano L, et al. Impact of BCR-ABL mutations on response to dasatinib after imatinib failure in elderly patients with chronic-phase chronic myeloid leukemia. Ann Hematol. 2013;92(2):179-183.

29. Pinilla-Ibarz J, Cortes J, Mauro MJ. Intolerance to tyrosine kinase inhibitors in chronic myeloid leukemia: definitions and clinical implications. Cancer. 2011;117(4):688-697.

30. Hochhaus A, Baccarani M, Deininger M, et al. Dasatinib induces durable cytogenetic responses in patients with chronic myelogenous leukemia in chronic phase with resistance or intolerance to imatinib. Leukemia. 2008;22(6):1200-1206.

31. Kantarjian H, Pasquini R, Lévy V, et al. Dasatinib or high-dose imatinib for chronic-phase chronic myeloid leukemia resistant to imatinib at a dose of 400 to 600 milligrams daily: two-year follow-up of a randomized phase 2 study (START-R). Cancer. 2009;115(18):4136-4147.

32. Shah NP, Kantarjian HM, Kim DW, et al. Intermittant target inhibition with dasatinib $100 \mathrm{mg}$ once daily preserves efficacy and improves tolerability in imatinib-resistant and -intolerant chronic-phase chronic myeloid leukemia. J Clin Oncol. 2008;26(19):3204-3212.

33. Valent P, Hadzijusufovic E, Schernthaner GH, Wolf D, Rea D, le Coutre P. Vascular safety issues in CML patients treated with BCR/ABL1 kinase inhibitors. Blood. 2015;125(6):901-906.

34. O'Hare T, Walters DK, Stoffregen EP, et al. In vitro activity of Bcr-Abl inhibitors AMN107 and BMS-354825 against clinically relevant imatinib-resistant Abl kinase domain mutants. Cancer Res. 2005; 65(11):4500-4505.

35. Quintas-Cardama A, Kantarjian H, O’Brien S, et al. Pleural effusion in patients with chronic myelogenous leukemia treated with dasatinib after imatinib failure. J Clin Oncol. 2007;25(25):3908-3914.

36. Porkka K, Khoury HJ, Paquette RL, Matloub Y, Sinha R, Cortes JE. Dasatinib $100 \mathrm{mg}$ once daily minimizes the occurrence of pleural effusion in patients with chronic myeloid leukemia in chronic phase and efficacy is unaffected in patients who develop pleural effusion. Cancer. 2010;116(2):377-386.

37. Eskazan AE, Eyice D, Kurt EA, et al. Chronic myeloid leukemia patients who develop grade I/II pleural effusion under second-line dasatinib have better responses and outcomes than patients without pleural effusion. Leuk Res. 2014;38(7):781-787.

38. Eskazan AE, Soysal T, Ongoren S, Gulturk E, Ferhanoglu B, Aydin Y. Pleural and pericardial effusions in chronic myeloid leukemia patients receiving low-dose dasatinib therapy. Haematologica. 2011; 96(3):e15

39. Quintás-Cardama A, Kantarjian H, Cortes J. Nilotinib-associated vascular events. Clin Lymphoma Myeloma Leuk. 2012;12(5):337-340.

40. Aichberger KJ, Herndlhofer S, Schernthaner GH, et al. Progressive peripheral arterial occlusive disease and other vascular events during nilotinib therapy in CML. Am J Hematol. 2011;86(7):533-539.

41. Gora-Tybor J, Medras E, Calbecka M, et al. Real-life comparison of severe vascular events and other non-hematological complications in patients with chronic myeloid leukemia undergoing second-line nilotinib or dasatinib treatment. Leuk Lymphoma. 2015;56(8):2309-2314.

42. Douxfils J, Haguet H, Mullier F, et al. Association between BCRABL tyrosine kinase inhibitors for chronic myeloid leukemia and cardiovascular events, major molecular response, and overall survival: a systematic review and meta-analysis. JAMA Oncol. Epub February 4, 2016. doi:10.1001/jamaoncol.2015.5932.
43. Dahlén T, Edgren G, Lambe M, et al; Swedish CML Group and the Swedish CML Register Group. Cardiovascular events associated with use of tyrosine kinase inhibitors in chronic myeloid leukemia: a population-based Cohort study. Ann Intern Med. Epub June 14, 2016. doi:10.7326/M15-2306.

44. Shah NP, Wallis N, Farber HW, et al. Clinical features of pulmonary arterial hypertension in patients receiving dasatinib. Am J Hematol. 2015;90(11):1060-1064.

45. Ozgur Yurttas N, Sadri S, Keskin D, et al. Real-life data and a single center experience on dasatinib-induced pulmonary arterial hypertension in patients with Philadelphia chromosome-positive leukemias. Blood. 2015;126(23):4037.

46. Peacock AJ, Murphy NF, McMurray JJ, Caballero L, Stewart S. An epidemiological study of pulmonary arterial hypertension. Eur Respir J. 2007;30(1):104-109.

47. Guignabert C, Montani D. Key roles of Src family tyrosine kinases in the integrity of the pulmonary vascular bed. Eur Respir J. 2013;41(1): 3-4.

48. Kim DH, Kamel-Reid S, Chang H, et al. Natural killer or natural killer/ $\mathrm{T}$ cell lineage large granular lymphocytosis associated with dasatinib therapy for Philadelphia chromosome positive leukemia. Haematologica. 2009;94(1):135-139.

49. Mustjoki S, Ekblom M, Arstila TP, et al. Clonal expansion of T/NKcells during tyrosine kinase inhibitor dasatinib therapy. Leukemia. 2009; 23(8):1398-1405.

50. Nagata Y, Ohashi K, Fukuda S, Kamata N, Akiyama H, Sakamaki H. Clinical features of dasatinib-induced large granular lymphocytosis and pleural effusion. Int J Hematol. 2010;91(5):799-807.

51. Paydas S. Dasatinib, large granular lymphocytosis, and pleural effusion: useful or adverse effect? Crit Rev Oncol Hematol. 2014;89(2): 242-247.

52. Qiu ZY, Xu W, Li JY. Large granular lymphocytosis during dasatinib therapy. Cancer Biol Ther. 2014;15(3):247-255.

53. Tanaka H, Nakashima S, Usuda M. Rapid and sustained increase of large granular lymphocytes and rare cytomegalovirus reactivation during dasatinib treatment in chronic myelogenous leukemia patients. Int J Hematol. 2012;96(3):308-319.

54. Kreutzman A, Jaatinen T, Greco D, et al. Killer-cell immunoglobulin-like receptor gene profile predicts good molecular response to dasatinib therapy in chronic myeloid leukemia. Exp Hematol. 2012; 40(11):906-913.e1.

55. Roux C, Nicolini FE, Rea D, et al. Reversible lymph node follicular hyperplasia associated with dasatinib treatment of chronic myeloid leukemia in chronic phase. Blood. 2013;122(17):3082-3084.

56. Ozawa MG, Ewalt MD, Gratzinger D. Dasatinib-related follicular hyperplasia: an underrecognized entity with characteristic morphology. Am J Surg Pathol. 2015;39(10):1363-1369.

57. Quintás-Cardama A, Kantarjian H, Ravandi F, et al. Bleeding diathesis in patients with chronic myelogenous leukemia receiving dasatinib therapy. Cancer. 2009;115(11):2482-2490.

58. Quintás-Cardama A, Han X, Kantarjian H, Cortes J. Tyrosine kinase inhibitor-induced platelet dysfunction in patients with chronic myeloid leukemia. Blood. 2009;114(2):261-263.

59. Eskazan AE, Hatemi İ, Öngören Aydın S, Ar MC, Soysal T. Acute colitis presenting with hematochezia in a patient with chronic myeloid leukemia during dasatinib therapy. Turk J Gastroenterol. 2014; 25(Suppl 1):233.

60. Erkut M, Erkut N, Ersoz S, Arslan M, Sonmez M. A case of acute colitis with severe rectal bleeding in a patient with chronic myeloid leukemia after dasatinib use. Acta Haematol. 2010;123(4):205-206. 


\section{Publish your work in this journal}

Drug Design, Development and Therapy is an international, peerreviewed open-access journal that spans the spectrum of drug design and development through to clinical applications. Clinical outcomes, patient safety, and programs for the development and effective, safe, and sustained use of medicines are the features of the journal, which has also been accepted for indexing on PubMed Central. The manuscript management system is completely online and includes a very quick and fair peer-review system, which is all easy to use. Visit http://www.dovepress.com/testimonials.php to read real quotes from published authors.

Submit your manuscript here: http://www.dovepress.com/drug-design-development-and-therapy-journal 